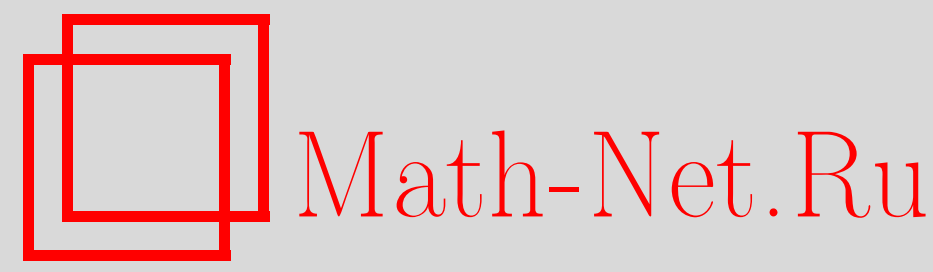

Ю. В. Муранов, Д. Реповш, Ф. Спаггиари, Перестройка троек многообразий, Матем. сб., 2003, том 194, номер 8, 139-160

DOI: https://doi.org/10.4213/sm764

Использование Общероссийского математического портала Math-Net.Ru подразумевает, что вы прочитали и согласны с пользовательским соглашением

http://www.mathnet.ru/rus/agreement

Параметры загрузки:

IP: 54.205 .225 .156

26 апреля 2023 г., 18:00:50 
УДК $513.8+515.1$

\author{
Ю.В. Муранов, Д. Реповш, $\Phi$. Спаггиари
}

\title{
Перестройка троек многообразий
}

\begin{abstract}
Группы препятствий к перестройкам пары многообразий были введены Уоллом для изучения задачи перестройки многообразия с подмногообразием. Эти группы тесно связаны с задачей расщепления гомотопической эквивалентности вдоль подмногообразия, и они используются во многих топологических и геометрических приложениях.

В работе вводится понятие перестройки тройки многообразий и описьваются алгебраические и геометрические свойства соответствующих групп препятствий. Затем показано, что эти группы тесно связаны с нормальньми инвариантами и классическими группами препятствий к перестройкам и расщеплениям данного многообразия. В частном случае односторонних подмногообразий получены связи между введенньми группами и спектральной последовательностью в теории перестроек, построенной Хэмблтоном и Харшиладзе.

Библиография: 25 названий.
\end{abstract}

\section{§1. Введение}

Пусть $X^{n}$ - замкнутое топологическое $n$-мерное многообразие с фундаментальной группой $\pi_{1}(X)$ и гомоморфизмом ориентации $w: \pi_{1}(X) \rightarrow\{ \pm 1\}$. Основной вопрос алгебраической и геометрической топологии состоит в описании всех возможных замкнутых топологических (кусочно линейных гладких) многообразий, которые (просто) гомотопически эквивалентны $X$ (см., например, [1]-[3]).

В настоящей работе мы будем рассматривать топологические многообразия и простые гомотопические эквивалентности между ними (см. [1], [4]).

Две простые гомотопические эквивалентности $f_{i}: M_{i} \rightarrow X, i=0,1$, называются әквивалентными, если существует такой сохраняющий ориентацию гомеоморфизм $g: M_{0} \rightarrow M_{1}$, что композиция $f_{1} \circ g$ гомотопна $f_{0}$. Пусть $\mathscr{S}^{T O P}(X)$ обозначает множество таких классов эквивалентности. Элементы этого множества называются гомотопическими триангуляииями (s-триангуляииями) многообразия $X$. Теория Раницкого о полном препятствии к перестройке (см. [1], [4]) дает отображение спектров

$$
X_{+} \wedge \mathbf{L}_{\bullet} \rightarrow \mathbb{L}\left(\pi_{1}(X)\right),
$$

где $\mathbb{L}\left(\pi_{1}(X)\right)$ обозначает $L$-спектр теории перестроек для фундаментальной группы $\pi_{1}(X)$ с

$$
\pi_{n}\left(\mathbb{L}\left(\pi_{1}(X)\right)\right) \cong L_{n}\left(\pi_{1}(X)\right)
$$

и $\mathbf{L} \bullet$ обозначает односвязное накрытие $\Omega$-спектра $\mathbb{L}(\mathbb{Z})$ с $\mathbf{L}_{\bullet 0} \simeq G / T O P$.

Работа вьполнена при поддержке Российского фонда фундаментальных исследований (грант № 02-01-00014), Министерства образования, науки и спорта Республики Словения (исследовательская программа № 101-509), Национального исследовательского совета и MURST Италии.

(C) Ю. В. Муранов, Д. Реповш, Ф. СпАГГИАри 2003 
Пусть $\mathbb{S}$ - гомотопический кослой отображения в (1). Для гомотопических групп $\mathscr{S}_{m}(X)=\pi_{m}(\mathbb{S})$ мы имеем изоморфизм $\mathscr{S}_{n+1}(X) \cong \mathscr{S}^{T O P}(X)$. Алгебраическая точная последовательность теории перестроек для многообразия $X$ (см. [1], [4])

$$
\cdots \rightarrow L_{m+1}\left(\pi_{1}(X)\right) \rightarrow \mathscr{S}_{m+1}(X) \rightarrow H_{m}\left(X ; \mathbf{L}_{\bullet}\right) \stackrel{\sigma}{\rightarrow} L_{m}\left(\pi_{1}(X)\right) \rightarrow \cdots
$$

является гомотопической длинной точной последовательностью расслоения в (1). Мы имеем изоморфизм $H_{n}\left(X_{+} ; \mathbf{L}_{\bullet}\right) \cong[X, G / T O P]$. Множество нормальных инвариантов $[X, G / T O P]$ состоит из классов нормальных кобордизмов нормальных отображений в многообразие $X$, и отображение ассамблеи $\sigma$ задается взятием препятствия к перестройке нормального отображения (см. [2]-[4]). Изучение отображения ассамблеи тесно связано со многими проблемами топологии многообразий (см., например, [3], [5]).

Пусть $Y \subset X-$ подмногообразие коразмерности $q$ в $X$. В этом случае отображение $\sigma$ разлагается в композицию

$$
[X, G / T O P] \rightarrow L P_{n-q}(F) \rightarrow L_{n}\left(\pi_{1}(X)\right)
$$

где $L P_{n-q}(F)$ - группа препятствий к перестройкам пары многообразий (см. [1], [3]). Группа $L P_{n-q}(F)$ функториально зависит от квадрата фундаментальных групп с ориентацией

$$
F=\left(\begin{array}{ccc}
\pi_{1}(\partial U) \longrightarrow & \pi_{1}(X \backslash Y) \\
\downarrow & & \downarrow \\
\pi_{1}(Y) \longrightarrow & \pi_{1}(X)
\end{array}\right)
$$

где $\partial U$ - граница трубчатой окрестности $Y$ в $X$. Группы $L P_{n-q}(F)$ тесно связаны с другими алгебраическими объектами, появляющимися в $L$-теории, посредством различных типов диаграмм точных последовательностей (см. [1], [3], [6]). В частности, эти групшы входят в следующую коммутативную диаграмму:

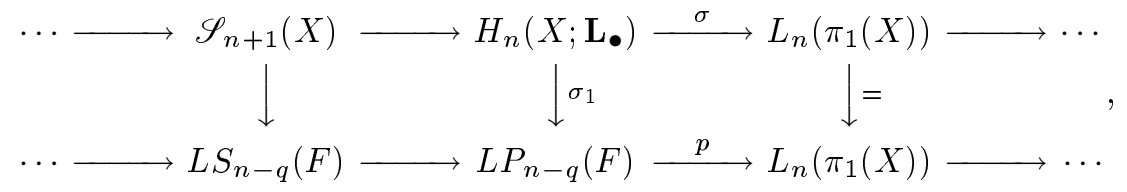

в которой все строки являются точными последовательностями и $L S_{n-q}(F)$ обозначает группу препятствий к расщеплению. Нижняя точная последовательность дает дополнительную информацию об отображении ассамблеи. В некоторых частных случаях эта последовательность применялась для исследования проблемы реализации элементов групп Уолла нормальными отображениями замкнутых многообразий и для построения спектральной последовательности Хэмблтона и Харшиладзе в теории перестроек (см., например, [7]-[9]). В случае односторонних подмногообразий имеется много результатов о связи групп $L S_{*}$ и $L P_{*}$ с классическими $L_{*}$-группами [6], [10]-[16].

Пусть $Z \subset Y \subset X$ - тройка замкнутых топологических многообразий, так что $n$ - размерность $X, q$-коразмерность $Y$ в $X$ и $q^{\prime}-$ коразмерность $Z$ в $Y$. 
Мы будем предполагать, что для каждой пары многообразий задано нормальное расслоение к подмногообразию (см. $[1 ; \S 7.2])$. В настоящей работе мы вводим групшы $L T_{n-q-q^{\prime}}(X, Y, Z)$, которые содержат препятствия к перестройкам тройки многообразий. Предположим, что $n-q-q^{\prime} \geqslant 5$. Для любого нормального отображения $(f, b) \in[X, G / T O P]$, где $f: M \rightarrow X$, мы определяем препятствие $\Theta_{*}(f, b) \in L T_{n-q-q^{\prime}}(X, Y, Z)$, которое тривиально тогда и только тогда, когда $(f, b)$ нормально кобордантно некоторой $s$-триангуляции тройки $(X, Y, Z)$. Понятие $s$-триангуляции для тройки многообразий (см. §3) является естественным обобщением $s$-триангуляции для пары многообразий, определенной в [1].

Отображение $\Theta_{*}$ входит в следуюшее естественное разложение отображений $\sigma_{1}$ и $\sigma$ :

$$
[X, G / T O P] \rightarrow L T_{n-q-q^{\prime}}(X, Y, Z) \rightarrow L P_{n-q}(F) \rightarrow L_{n}\left(\pi_{1}(X)\right)
$$

Группы $L T_{*}$ тесно связаны с точной последовательностью теории перестроек (2) и с группами препятствий к перестройкам и расщеплению для пар многообразий $Y \subset X$ и $Z \subset Y$. Эти связи определяются некоторыми косами точных последовательностей, которые содержат классические групшы препятствий.

Пусть $(X, Y, Z)$ - тройка многообразий, для которой $(X, Y)$ и $(Y, Z)$ являются парами Браудера-Ливси (см. [7], [17], [18]). Для такой тройки [19] Хэмблтон и Педерсен ввели группы $L N S_{*}$, которые связаны с группами препятствий к расщеплению и $L_{*}$-группами в этом случае. Группы $L T_{*}$ также связаны с итерированными трансферами и с $L N S_{*}$-группами (см. [20]).

Для доказательства наших основных результатов мы будем использовать $L$-спектры и реализацию естественных отображений в $L$-теории на уровне спектров (см. [3], [4], [6], [21]-[24]).

Работа организована следующим образом. В $\S 2$ мы напоминаем необходимые определения и факты о $L$-спектрах и их связях с теорией перестроек. Далее, мы доказываем несколько технических лемм. В $\S 3$ мы определяем спектр $\mathbb{L} T$ с гомотопическими группами $\pi_{n}(\mathbb{L} T)=L T_{n}(X, Y, Z)$ и доказываем основную теорему работы. Затем мы получаем некоторые косы точных последовательностей, которые содержат групшы $L T_{n}(X, Y, Z)$ и классические группы препятствий к перестройкам и расщеплениям. В $\S 4$ мы описываем некоторые применения спектра $\mathbb{L} T$ и групп $L T_{n}(X, Y, Z)$ к случаю односторонних подмногообразий и их связи со спектральной последовательностью в теории перестроек, построенной Хэмблтоном и Харшиладзе в [8].

\section{$\S 2$. Спектры в $L$-теории}

В этом параграфе мы напомним некоторые основные определения и известные факты о реализации алгебраических объектов и естественных отображений в теории перестроек на уровне спектров (см. [1], [3], [4], [11], [21]-[25]). Затем мы доказываем две технические леммы.

Топологическое нормальное отображение $(f, b): M \rightarrow X$ в замкнутое топологическое $n$-мерное многообразие $X(t$-триангуляиия $X)$ определяется следуюшими условиями (см. [1], [4]):

i) замкнутьм топологическим $n$-мерным многообразием $M$ с нормальным 
блочным топологическим расслоением

$$
\begin{gathered}
\nu_{M}=\nu_{M \subset S^{n+k}}: M \rightarrow B T O P(k), \\
\rho_{M}: S^{n+k} \rightarrow S^{n+k} / \overline{S^{n+k} \backslash E\left(\nu_{M}\right)}=T\left(\nu_{M}\right) ;
\end{gathered}
$$

ii) нормальным блочным топологическим расслоением над многообразием $X$

$$
\begin{aligned}
& \nu_{X}: X \rightarrow B T O P(k), \\
& \rho_{X}: S^{n+k} \rightarrow T\left(\nu_{X}\right) ;
\end{aligned}
$$

iii) отображением степени один $f: M \rightarrow X$ с отображением $b: \nu_{M} \rightarrow \nu_{X}$ блочных топологических расслоений, которое накрывает $f$. Более того, мы имеem

$$
T(b)_{*}\left(\rho_{M}\right)=\rho_{X} \in \pi_{n+k}\left(T\left(\nu_{X}\right)\right) .
$$

Множество топологических нормальных инвариантов многообразия $X$ состоит из всех классов конкордантности топологических нормальных отображений $(f, b): M \rightarrow X$. В соответствии с [1] для любого $n \geqslant 5$ это множество совпадает с множеством $[X, G / T O P]$.

Пусть $Y \subset X$ - замкнутое подмногообразие коразмерности $q$ в замкнутом топологическом многообразии $X$. В нашей работе мы будем рассматривать только пары многообразий $(X, Y, \xi)$ в смысле Раницкого (см. $[1 ; \S 7.2])$. Это значит, что $Y$ является локально плоским подмногообразием и нормальное расслоение

$$
\xi=\xi_{Y \subset X}: Y \rightarrow \widehat{\operatorname{BTOP}}(q)
$$

таково, что

$$
X=E(\xi) \bigcup_{S(\xi)} \overline{X \backslash E(\xi)} .
$$

В частности, ассоциированное $\left(D^{q}, S^{q-1}\right)$ расслоение

$$
\left(D^{q}, S^{q-1}\right) \rightarrow(E(\xi), S(\xi)) \rightarrow Y
$$

определено.

Топологическое нормальное отображение $(t$-триангуляция $(X, Y, \xi))$ задается следующим объектом (подробнее см. в [1]):

$$
((f, b),(g, c)):(M, N) \rightarrow(X, Y) .
$$

Здесь $(f, b)$ является $t$-триангуляцией многообразия $X$, для которой $f$ трансверсально $Y$ с $N=f^{-1}(Y)$, и $(M, N)$ является топологической парой многообразий с нормальным расслоением

$$
\nu: N \stackrel{\left.f\right|_{N}}{\longrightarrow} Y \stackrel{\xi}{\longrightarrow} \widehat{\operatorname{BTOP}}(q) .
$$

Ограничение

$$
\left.(f, b)\right|_{N}=(g, c): N \rightarrow Y
$$


является $t$-триангуляцией $Y$. Ограничение

$$
\left.(f, b)\right|_{P}=(h, d):(P, S(\nu)) \rightarrow(Z, S(\xi))
$$

является $t$-триангуляцией $(Z, S(\xi))$, где

$$
P=\overline{M \backslash E(\nu)}, \quad Z=\overline{X \backslash E(\xi)} .
$$

Ограничение

$$
\left.(h, d)\right|_{S(\nu)}: S(\nu) \rightarrow S(\xi)
$$

совпадает с индуцированным отображением

$$
(g, c)^{!}: S(\nu) \rightarrow S(\xi)
$$

и $(f, b)=(g, c)^{!} \cup(h, d)$.

В соответствии с [1] множество классов конкордантности $t$-триангуляций $(X, Y, \xi)$ совпадает с множеством классов $t$-триангуляций многообразия $X$.

Некоторая $t$-триангуляция

$$
((f, b),(g, c)):(M, N) \rightarrow(X, Y)
$$

пары многообразий $(X, Y, \xi)$ задает $s$-триангуляцию этой пары (см. [1]), если отображения

$$
f: M \rightarrow X, \quad g: N \rightarrow Y, \quad h:(P, S(\nu)) \rightarrow(Z, S(\xi))
$$

являются $s$-триангуляциями.

Далее мы будем всегда предполагать, что $n-q \geqslant 5$. Простая гомотопическая эквивалентность $f: M \rightarrow X$ расшепляется вдоль подмногообразия $Y$, если она гомотопически эквивалентна отображению $g$, которое удовлетворяет свойствам (5). То есть отображение $g$ является $s$-триангуляцией $(X, Y, \xi)$. Сушествует группа $L S_{n-q}(F)$ препятствий к расшеплению простой гомотопической эквивалентности. Эта группа зависит только от $n-q(\bmod 4)$ и универсально-отталкивающего квадрата $F$ фундаментальных групп с ориентацией (3) (см. [1]). Рассмотрим нормальное отображение $(f, b) \in[X, G / T O P]$ с $f: M \rightarrow X$. Тогда согласно [1] существует группа $L P_{n-q}(F)$ препятствий к перестройкам пары многообразий $(X, Y)$, которая зависит также только от $n-q(\bmod 4)$ и квадрата $F$. Препятствие $\sigma_{1}(f, b) \in$ $L P_{n-q}(F)$ к перестройке нормального отображения в пару $(X, Y)$ будет тривиально тогда и только тогда, когда класс конкордантности $(f, b)$ содержит $s$-триангуляцию пары $(X, Y, \xi)$.

Верхняя строка в диаграмме (4) является точной последовательностью в теории перестроек для многообразия $X$, а нижняя строка - точная последовательность, содержащая групшы $L S_{*}(F), L P_{*}(F)$ и $L_{*}\left(\pi_{1}(X)\right)$. Глубокие связи между этими группами даются следующей косой точных последовательностей (см. [1] и [3]):

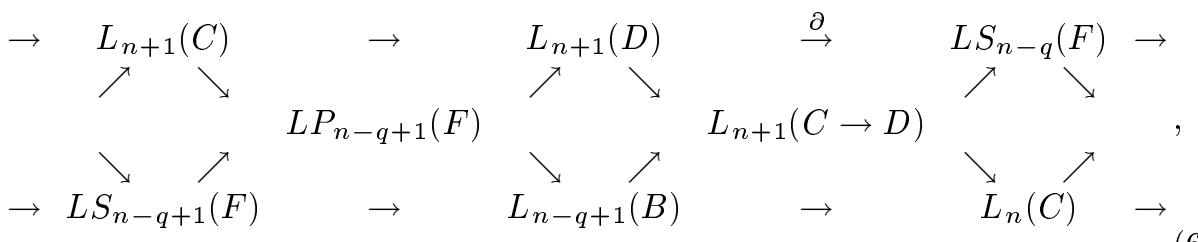


где $A=\pi_{1}(\partial U), B=\pi_{1}(Y), C=\pi_{1}(X \backslash Y)$ и $D=\pi_{1}(X)$. Отображение

$$
\partial: L_{n+1}\left(\pi_{1}(X)\right) \rightarrow L S_{n-q}(F)
$$

в диаграмме (6) совпадает с композицией

$$
L_{n+1}\left(\pi_{1}(X)\right) \rightarrow \mathscr{S}_{n+1}(X) \rightarrow L S_{n-q}(F)
$$

возникающей из диаграммы (4).

Пусть $\mathscr{S}_{n+1}(X, Y, \xi)$ - множество классов конкордантности $s$-триангуляций пары многообразий $(X, Y, \xi)($ см. [1]).

Из [17] следует, что сушествует коммутативная диаграмма

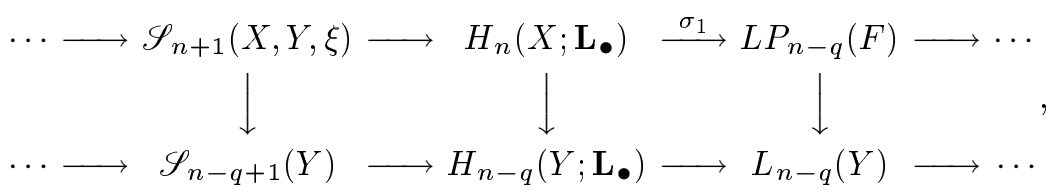

строки которой - точные последовательности. Нижняя строка является алгебраической точной последовательностью теории перестроек для многообразия $Y$. Заметим, что множество $\mathscr{S}_{n+1}(X, Y, \xi)$ имеет групповую структуру.

Теперь мы напомним некоторые необходимые определения и свойства, касающиеся гомотопической категории спектров (см., например, [25]). Cneкmp $\mathbb{E}$ состоит из семейства $C W$-комплексов $\left\{\left(E_{n}, *\right)\right\}, n \in \mathbb{Z}$, с семейством клеточных отображений $\left\{\varepsilon_{n}: S E_{n} \rightarrow E_{n+1}\right\}$, где $S E_{n}$ является надстройкой пространства $E_{n}$.

Для каждого отображения $\varepsilon_{n}$ сушествует сопряженное отображение $\varepsilon_{n}^{\prime}: E_{n} \rightarrow$ $\Omega E_{n+1}$. Спектр $\mathbb{E}$ называется $\Omega$-спектром, если все сопряженные отображения являются гомотопическими эквивалентностями.

Напомним определение спектра $\Sigma \mathbb{E}$ с $\{\Sigma \mathbb{E}\}_{n}=\mathbb{E}_{n+1}$ и $\{\Sigma \varepsilon\}_{n}=\varepsilon_{n+1}$. Легко видеть, что функтор $\Sigma$ имеет обратный функтор $\Sigma^{-1}$. Таким образом, можно определить итерированные функторы $\Sigma^{k}, k \in \mathbb{Z}$, на категории спектров. Имеет место изоморфизм гомотопических групп $\pi_{n}(\mathbb{E})=\pi_{n+k}\left(\Sigma^{k} \mathbb{E}\right)$ для любого спектpa $\mathbb{E}$ (cм. [25]).

В гомотопической категории спектров понятия универсально-отталкивающего и универсально-притягивающего квадрата эквивалентны. Таким образом, гомотопически коммутативньй квадрат спектров

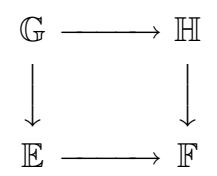

является универсально-притягивающим (универсально-отталкивающим) квадратом тогда и только тогда, когда слои (кослои) двух горизонтальных или двух вертикальных отображений естественно гомотопически эквивалентны (см., например, [8]).

Пусть $f: \pi \rightarrow \pi^{\prime}$ - гомоморфизм ориентированных групп. Тогда может быть определено расслоение $\Omega$-спектров

$$
\mathbb{L}(\pi) \rightarrow \mathbb{L}\left(\pi^{\prime}\right) \rightarrow \mathbb{L}(f),
$$


как показано, например, в [21]. Здесь мы имеем $\pi_{n}(\mathbb{L}(\pi))=L_{n}(\pi)$ и аналогичные соотношения для других спектров. Гомотопическая длинная точная последовательность корасслоения (8) дает относительную точную последовательность $L$-групп для отображения $f$

$$
\cdots \rightarrow L_{n}(\pi) \rightarrow L_{n}\left(\pi^{\prime}\right) \rightarrow L_{n}(f) \rightarrow L_{n-1}(\pi) \rightarrow \cdots
$$

Пусть $X$ - замкнутое $n$-мерное топологическое многообразие и $p: E \rightarrow X-$ расслоение со слоем $M^{m}$ ( $M^{m}-m$-мерное замкнутое топологическое многообразие). Тогда отображение трансфера

$$
p^{*}: L_{n}\left(\pi_{1}(X)\right) \rightarrow L_{n+m}\left(\pi_{1}(E)\right)
$$

может быть определено (см. [3], [23], [24]). Это отображение реализуется на уровне спектров отображением $\Omega$-спектров

$$
p^{!}: \mathbb{L}\left(\pi_{1}(X)\right) \rightarrow \Sigma^{-m} \mathbb{L}\left(\pi_{1}(E)\right) .
$$

Для пары многообразий $(X, Y)$ рассмотрим гомотопически коммутативную диаграмму спектров

$$
\mathbb{L}\left(\pi_{1}(Y)\right) \underset{p^{!} \searrow}{\stackrel{p_{1}^{!}}{\rightarrow}} \begin{array}{cccc}
\Sigma^{-q} \mathbb{L}\left(\pi_{1}(\partial U) \rightarrow \pi_{1}(U)\right) & \stackrel{\alpha}{\rightarrow} & \Sigma^{-q} \mathbb{L}\left(\pi_{1}(X \backslash Y) \rightarrow \pi_{1}(X)\right) \\
& \Sigma^{1-q} \mathbb{L}\left(\pi_{1}(\partial U)\right) & \stackrel{\beta}{\rightarrow} & \Sigma^{1-q} \mathbb{L}\left(\pi_{1}(X \backslash Y)\right)
\end{array},
$$

где левые отображения являются отображениями трансфера, а правые горизонтальные отображения индуцированы горизонтальными отображениями квадрата $F$.

Определим спектр $\mathbb{L} S(F)$ как гомотопический кослой отображения

$$
\Sigma^{-1}\left(\alpha p_{1}^{!}\right): \Sigma \mathbb{L}\left(\pi_{1}(Y)\right) \rightarrow \Sigma^{-q-1} \mathbb{L}\left(\pi_{1}(X \backslash Y) \rightarrow \pi_{1}(X)\right)
$$

и спектр $\mathbb{L} P(F)$ как гомотопический кослой отображения

$$
\Sigma^{-1}\left(\beta p^{!}\right): \Sigma^{-1} \mathbb{L}\left(\pi_{1}(Y)\right) \rightarrow \Sigma^{-q} \mathbb{L}\left(\pi_{1}(X \backslash Y)\right)
$$

(см. подробнее [10], [11], [14], [22]). Пусть $L S_{n}(F)$ и $L P_{n}(F)$ - групшы препятствий к расщеплению и групшы препятствий к перестройке пары многообразий $(X, Y)$ соответственно. Тогда имеют место изоморфизмы

$$
\pi_{n}(\mathbb{L} S(F)) \cong L S_{n}(F), \quad \pi_{n}(\mathbb{L} P(F)) \cong L P_{n}(F)
$$

Используя эти определения и гомотопически коммутативную диаграмму спектров (9), мы получаем следующую гомотопически коммутативную диаграмму спектров

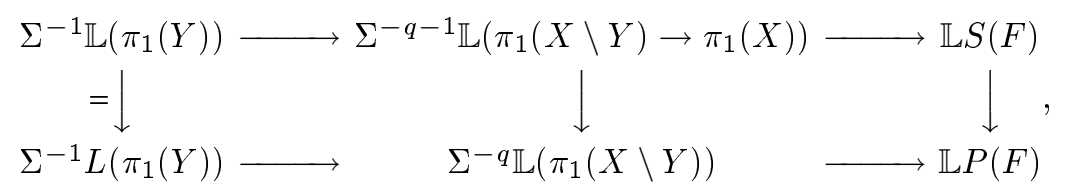


в которой правый квадрат является универсально-отталкивающим квадратом. Гомотопические длинные точные последовательности отображений из правого квадрата дают коммутативную диаграмму (6).

Пусть $Y^{n-q} \subset X^{n}$ - замкнутое подмногообразие коразмерности $q$ в $n$-мерном замкнутом топологическом многообразии $X$. Тогда коммутативная диаграмма (4) определена.

ЛЕмма 1. В приведенных выше обозначениях имеет место такой гомотопически коммутативный квадрат спектров

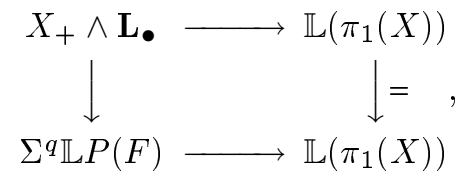

что коммутативная диаграмма (4) получается индуиированным отображсением гомотопической длинной точной последовательности верхнего отображсения в (11) в соответствующую точную последовательность нижнего отображсения в (11).

ДокАЗАТЕЛЬСтво. Рассмотрим коммутативную диаграмму

$$
\begin{aligned}
H_{n-q}\left(Y ; \mathbb{L}_{\bullet}\right) \stackrel{\cong}{\cong} & H_{n}\left(X, X \backslash Y ; \mathbb{L}_{\bullet}\right) \\
\searrow & H_{n-1}\left(X \backslash Y ; \mathbb{L}_{\bullet}\right) \rightarrow H_{n-1}\left(X ; \mathbb{L}_{\bullet}\right)
\end{aligned},
$$

где верхнее горизонтальное отображение является трансфером. Вертикальное отображение и нижнее горизонтальное отображение происходят из гомологической длинной точной последовательности пары $(X, X \backslash Y)$. Таким образом, диаграмма (12) порождается следуюшей гомотопически коммутативной диаграммой спектров:

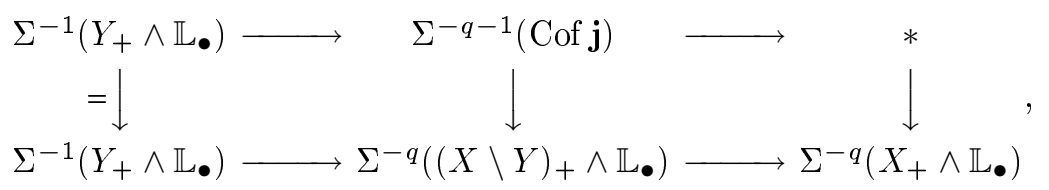

где Cof $\mathbf{j}$ - гомотопический кослой естественного вложения

$$
\mathbf{j}:(X \backslash Y)_{+} \wedge \mathbf{L}_{\bullet} \rightarrow X_{+} \wedge \mathbf{L}_{\bullet}
$$

Верхняя строка и вертикальные строки в диаграмме (13) являются корасслоениями. Кослои двух правых вертикальных отображений в (13) гомотопически эквивалентны $\Sigma^{-q}\left(X_{+} \wedge \mathbb{L}_{\bullet}\right)$. Кослои двух правых вертикальных отображений в (10) гомотопически эквивалентны $\Sigma^{-q} \mathbb{L}\left(\pi_{1}(X)\right)$. Отображение ассамблеи в (1) (см. [1]) индуцирует отображения спектров из левого квадрата диаграммы (13) в левьй квадрат диаграммы (10). По [25] мы получаем отображения спектров из правого столбца диаграммы (13) в правый столбец диаграммы (10). Таким образом, мы имеем гомотопически коммутативную диаграмму

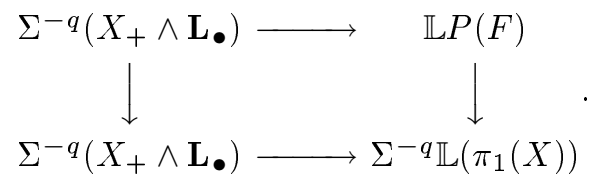

Применение функтора $\Sigma^{q}$ к этой диаграмме дает требуемый результат. 
ЛЕмма 2. Существует такой гомотопически коммутативный квадрат спектров

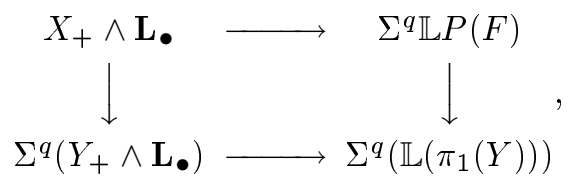

что коммутативная диаграмма (7) получается индуцированнылм отображением гомотопической длинной точной последовательности верхнего отображсения в (14) в соответствующую точную последовательность нижнего отображсения в (14).

Доказательство аналогично доказательству леммы 1.

\section{§3. Перестройки троек многообразий}

Пусть $Z \subset Y \subset X$ - такая тройка замкнутых топологических многообразий, что $n$ - размерность $X, q$-коразмерность $Y$ в $X$ и $q^{\prime}-$ коразмерность $Z$ в $Y$. В этом параграфе мы предполагаем, что для данной тройки заданы пары многообразий $(X, Y, \xi)$ и $\left(Y, Z, \xi_{1}\right)$. Иногда мы не будем записывать расслоения в обозначениях, если это не будет приводить к недоразумению. Мы предполагаем, что размерность $k$ многообразия $Z$ больше или равна 5 , т.е. $k=n-q-q^{\prime} \geqslant 5$.

ОПРЕДЕЛЕНИЕ 1. Топологическое нормальное отображение $(t$-триангуляиия $)$

$$
((f, b),(g, c),(h, d)):(M, N, K) \rightarrow(X, Y, Z)
$$

тройки многообразий $(X, Y, Z)$ состоит из $t$-триангуляций пар многообразий

$$
\begin{gathered}
((f, b),(g, c)):(M, N) \rightarrow(X, Y), \\
((g, c),(h, d)):(N, K) \rightarrow(Y, Z) .
\end{gathered}
$$

В частности, топологическое нормальное отображение

$$
(f, b): M \rightarrow X
$$

топологически трансверсально к $Y$ относительно $\xi$ с $N=f^{-1}(Y)$ и топологическое нормальное отображение

$$
(g, c): N \rightarrow Y
$$

топологически трансверсально к $Z$ относительно $\xi_{1}$ с $K=g^{-1}(Z)$.

ОПРЕДЕЛЕНИЕ 2. Назовем $t$-триангуляцию

$$
((f, b),(g, c),(h, d)):(M, N, K) \rightarrow(X, Y, Z)
$$

тройки $(X, Y, Z) s$-триангулячией, если составляющие $t$-триангуляции

$$
\begin{aligned}
& ((f, b),(g, c)):(M, N) \rightarrow(X, Y), \\
& ((g, c),(h, d)):(N, K) \rightarrow(Y, Z)
\end{aligned}
$$

являются $s$-триангуляциями. 
Рассмотрим топологическое нормальное отображение $(f, b) \in[X, G / T O P]$ с $f: M \rightarrow X$. Из топологической теоремы трансверсальности (см. [1]) следует, что $(f, b)$ задает некоторую $t$-триангуляцию

$$
((f, b),(g, c),(h, d)):(M, N, K) \rightarrow(X, Y, Z)
$$

тройки $(X, Y, Z)$. Мы будем говорить, что существует перестройка многообразия $M$ до получения $s$-триангуляции тройки $(X, Y, Z)$, если полученная $t$-триангуляция нормально кобордантна некоторой $s$-триангуляции тройки многообразий $(X, Y, Z)$.

Напомним, что множество классов нормальных кобордизмов нормальных отображений в данное замкнутое топологическое многообразие $X$ совпадает с множеством $[X, G / T O P]=H_{n}\left(X ; \mathbf{L}_{\bullet}\right)($ см. [1], [4]).

Квадрат $F$ в $(3)$ и группы препятствий $L P_{*}(F)$ и $L S_{*}(F)$ определены для пары многообразий $Y \subset X$. Обозначим через

$$
\Psi=\left(\begin{array}{ccc}
\pi_{1}(\partial V) \longrightarrow & \pi_{1}(Y \backslash Z) \\
\downarrow & & \downarrow \\
\pi_{1}(Z) \longrightarrow & \pi_{1}(Y)
\end{array}\right)
$$

квадрат фундаментальных групп с ориентациями, где $\partial V$ - граница трубчатой окрестности $Z$ в $Y$. Тогда групшы препятствий к перестройкам $L P_{*}(\Psi)$ и группы препятствий к расщеплению $L S_{*}(\Psi)$ определены для пары многообразий $(Y, Z)$.

Рассмотрим отображение

$$
v: L P_{n-q+1}(F) \rightarrow L S_{n-q-q^{\prime}}(\Psi)
$$

определяемое композицией

$$
L P_{n-q+1}(F) \rightarrow \mathscr{I}_{n+1}(X, Y, \xi) \rightarrow \mathscr{I}_{n-q+1}(Y) \rightarrow L S_{n-q-q^{\prime}}(\Psi)
$$

Два первых отображения композиции (16) лежат в диаграмме (7), а третье отображение $\mathscr{S}_{n-q+1}(Y) \rightarrow L S_{n-q-q^{\prime}}(\Psi)$ лежит в диаграмме, аналогичной диаграмме $(4)$, записанной для пары многообразий $(Y, Z)$.

ПРЕДЛОЖЕНИЕ 1. Отображение $v$ может быть реализовано отображением $\Omega$-спектров

$$
\mathbf{v}: \mathbb{L} P(F) \rightarrow \Sigma^{q^{\prime}+1} \mathbb{L} S(\Psi)
$$

так, что индуцированный гомоморфизм гомотопических групп

$$
\mathbf{v}_{*}: \pi_{n-q+1}(\mathbb{L} P(F))=L P_{n-q+1}(F) \rightarrow L S_{n-q-q^{\prime}}(\Psi)=\pi_{n-q+1}\left(\Sigma^{q^{\prime}+1} \mathbb{L} S(\Psi)\right)
$$

cовпадает c v. 
ДоказАТЕЛЬСтво. Обозначим через $\mathbb{S}(X, Y, \xi)$ гомотопический кослой отображения

$$
X_{+} \wedge \mathbf{L}_{\bullet} \rightarrow \Sigma^{q} \mathbb{L} P(F),
$$

рассмотренного в утверждении леммы 2. Тогда мы имеем

$$
\pi_{n+1}(\mathbb{S}(X, Y, \xi))=\mathscr{S}_{n+1}(X, Y, \xi)
$$

Композиция

$$
L P_{n-q+1}(F) \rightarrow \mathscr{S}_{n+1}(X, Y, \xi) \rightarrow \mathscr{S}_{n-q+1}(Y)
$$

отображений, возникающих из диаграммы (7), реализуется следующей композицией отображений спектров:

$$
\mathbb{L} P(F) \rightarrow \Sigma^{-q} \mathscr{S}_{n+1}(X, Y, \xi) \rightarrow \mathbb{S}(Y)
$$

по лемме 2 и [25]. Отображение $\mathscr{S}_{n-q+1}(Y) \rightarrow L S_{n-q-q^{\prime}}(\Psi)$ реализуется на уровне спектров по лемме 1 для пары многообразий $(Y, Z)$. Таким образом, отображение $v$ реализуется на уровне спектров, что и требовалось доказать.

Обозначим через $\mathbb{L} T(X, Y, Z)$ гомотопический кослой отображения $\Sigma^{-q^{\prime}-1} \mathbf{v}$ и через

$$
L T_{n}(X, Y, Z)=\pi_{n}(\mathbb{L} T(X, Y, Z))
$$

- гомотопические группы полученного спектра. В частности, мы имеем корасслоение $\Omega$-спектров

$$
\Sigma^{-q^{\prime}-1} \mathbb{L} P(F) \rightarrow \mathbb{L} S(\Psi) \rightarrow \mathbb{L} T(X, Y, Z) .
$$

Гомотопическая длинная точная последовательность корасслоения (17) дает следуюшую точную последовательность:

$$
\cdots \rightarrow L P_{n-q+1}(F) \rightarrow L S_{n-q-q^{\prime}}(\Psi) \rightarrow L T_{n-q-q^{\prime}}(X, Y, Z) \rightarrow \cdots
$$

Tеорема 1. Существует отображение спектров

$$
\Theta: X_{+} \wedge \mathbf{L}_{\bullet} \rightarrow \Sigma^{q+q^{\prime}} \mathbb{L} T(X, Y, Z)
$$

которое индуцирует гомоморфизм

$$
\Theta_{*}: H_{n}\left(X ; \mathbf{L}_{\bullet}\right) \rightarrow L T_{n-q-q^{\prime}}(X, Y, Z)
$$

гомотопических групп. Пусть $(f, b) \in[X, G / T O P]=H_{n}\left(X ; \mathbf{L}_{\bullet}\right)-$ топологическое нормальное отображсение с $f: M \rightarrow X$. Тогда $(f, b)$ нормально кобордантно некоторой s-триангуляции тройки $(X, Y, Z)$ тогда и только тогда, когда $\Theta_{*}(f, b)=0$. 
ДокАЗАТЕЛЬство. Рассмотрим диаграмму спектров

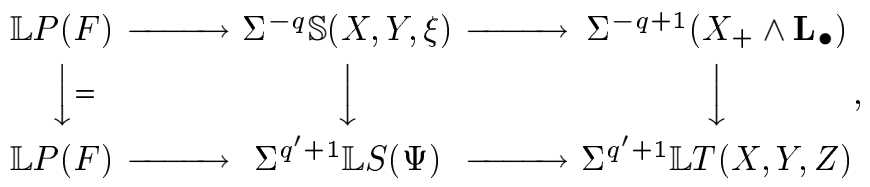

в которой левый квадрат существует по предложению 1 и горизонтальные строки являются корасслоениями. Следовательно, существует такое правое вертикальное отображение (см. [25]), что диаграмма (19) будет гомотопически коммутативна. Применение функтора $\Sigma^{q-1}$ к правому вертикальному отображению дает отображение $\Theta$. Заметим здесь, что правый квадрат диаграммы (19) является универсально-отталкивающим (и универсально-притягивающим), поскольку слои горизонтальных отображений естественно гомотопически эквивалентны. Рассмотрение гомотопических длинных точных последовательностей строк гомотопически коммутативной диаграммы (19) дает следующую коммутативную диаграмму:

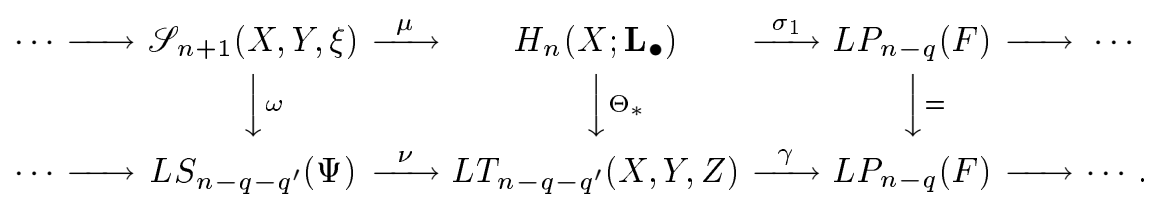

Предположим, что нормальное отображение $(f, b)$ нормально кобордантно $s$-триангуляции

$$
\left(\left(f^{\prime}, b^{\prime}\right),\left(g^{\prime}, c^{\prime}\right),\left(h^{\prime}, d^{\prime}\right)\right):(M, N, K) \rightarrow(X, Y, Z)
$$

Гомоморфизм $\Theta_{*}$ зависит только от класса нормального кобордизма $x=[(f, b)]=$ $\left[\left(f^{\prime}, b^{\prime}\right)\right] \in H_{n}\left(X ; \mathbf{L}_{\bullet}\right)$. По определению $s$-триангуляции тройки $(X, Y, Z)$ представитель $\left(\left(f^{\prime}, b^{\prime}\right),\left(g^{\prime}, c^{\prime}\right)\right)$ класса $x$ лежит в множестве $\mathscr{S}_{n+1}(X, Y, \xi)$, которое состоит из классов конкордантности $s$-триангуляций пары многообразий $(X, Y, \xi)[1]$. Обозначим через $y$ класс $\left(\left(f^{\prime}, b^{\prime}\right),\left(g^{\prime}, c^{\prime}\right)\right)$ в $\mathscr{S}_{n+1}(X, Y, \xi)$. Тогда $x=\mu(y)$. По определению $s$-триангуляции $\left(\left(f^{\prime}, b^{\prime}\right),\left(g^{\prime}, c^{\prime}\right),\left(h^{\prime}, d^{\prime}\right)\right)$ мы получаем, что простая гомотопическая эквивалентность $g^{\prime}: N \rightarrow Y$ уже расщеплена вдоль подмногообразия $Z$. Следовательно, по определению отображения $\omega$ мы имеем $\omega(y)=0 \in$ $L S_{n-q-q^{\prime}}(\Psi)$ и $\nu \omega(y)=0$. Тогда коммутативность диаграммы $(20)$ влечет

$$
\Theta_{*}(x)=\Theta_{*} \mu(y)=\nu \omega(y)=0 .
$$

Это доказывает первую импликацию.

Обратно, пусть $\Theta_{*}(x)=0$ для некоторого элемента $x=[(f, b)] \in H_{n}\left(X ; \mathbf{L}_{\bullet}\right)$. 
Рассмотрим следующую коммутативную диаграмму:

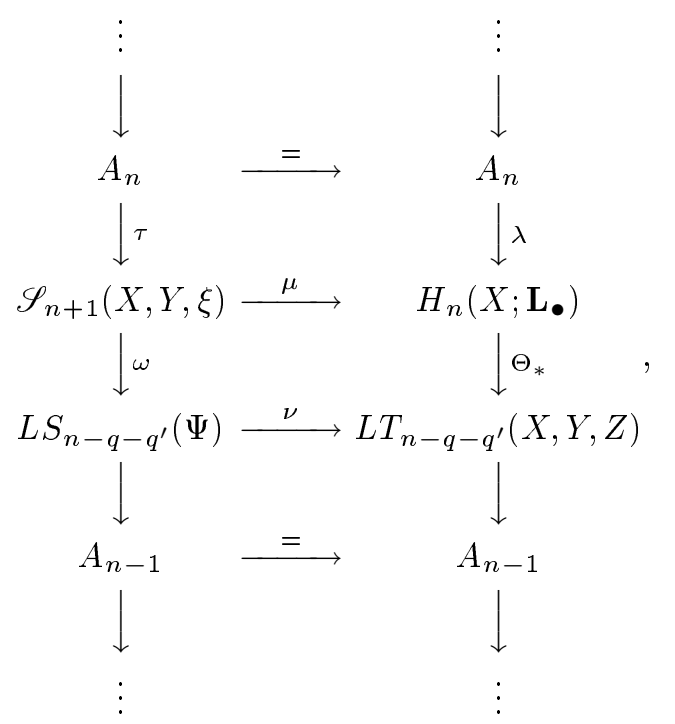

в которой вертикальные столбцы - точные последовательности. Диаграмма (21) следует из правого универсально-отталкивающего квадрата в (19). Здесь $A_{i}$ - гомотопические группы гомотопического кослоя вертикального отображения в правом квадрате диаграммы (19). Поскольку $\Theta_{*}(x)=0$, сушествует такой элемент $a \in A_{n}$, что $\lambda(a)=x$ и, следовательно, $x=\mu \tau(a)$. Класс $\tau(a) \in \mathscr{S}_{n+1}(X, Y, \xi)$ представляется $s$-триангуляцией

$$
((f, b),(g, c)):(M, N) \rightarrow(X, Y) .
$$

Поскольку $\omega \circ \tau=0$, то $s$-триангуляция $(g, c): N \rightarrow Y$ гомотопна $s$-триангуляции

$$
\left(\left(g_{1}, c_{1}\right),(h, d)\right):(N, K) \rightarrow(Y, Z) .
$$

Пусть $F: N \times I \rightarrow Y$ - некоторая гомотопия с $\left.F\right|_{N \times 0}=g,\left.F\right|_{N \times 1}=g_{1}$. По определению мы имеем трубчатую окрестность $Y$ в $X$ с нормальным расслоением $\xi$, для которой $g^{*}(\xi)$ будет трубчатой окрестностью $N$ в $M$. Мы можем записать индуцированное расслоение $F^{*}(\xi)$ над $N \times I$ как $g^{*}(\xi) \times I$. Следовательно, мы можем продолжить гомотопию $F$ посредством гомотопии $G: M \times I \rightarrow X$ c $G(x, t)=g(x)$ для таких $x \in M$, которые не лежат в трубчатой окрестности подмногообразия $N$. Ограничение $\left.G\right|_{M \times 1}$ дает $s$-триангуляцию тройки $(X, Y, Z)$.

ТЕОРема 2. В приведенных выше обозначениях имеет место коммутативная диаграмма точных последовательностей

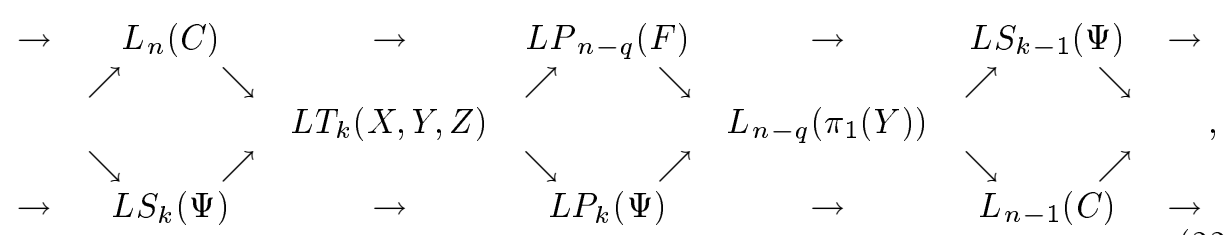

где $k=n-q-q^{\prime}-$ размерность подмногообразия $Z$ и $C=\pi_{1}(X \backslash Y)$. 
ДокАЗАТЕльство. Диаграмма (6) для пары многообразий $(X, Y)$ порождается следующим универсально-отталкивающим квадратом спектров [25] (см. также [6], [8] и [11] для случая односторонних подмногообразий):

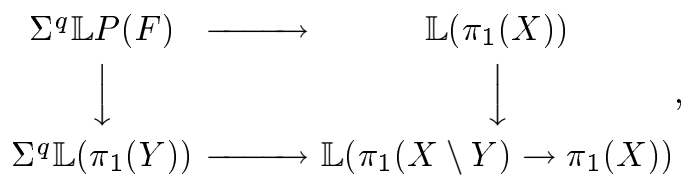

где верхнее горизонтальное отображение следует из диаграммы (11), а левое вертикальное отображение является отображением из диаграммы (14).

Для пары многообразий $(Y, Z)$ сушествует аналогичный универсально-отталкивающий квадрат спектров

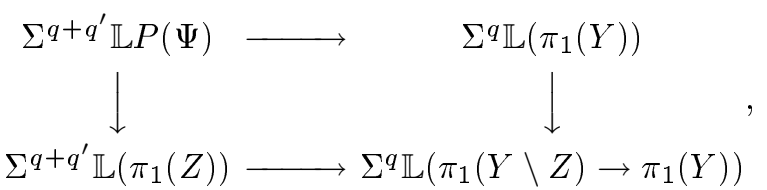

записанньй со сдвигом размерностей. Из диаграмм (23) и (24), используя определение спектра $\mathbb{L} T(X, Y, Z)$, получаем гомотопически коммутативную диаграмму спектров

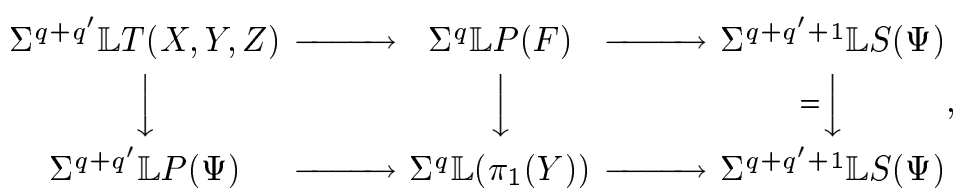

где существование левого отображения следует из [25]. Левый квадрат в диаграмме (25) является универсально-отталкивающим квадратом, поскольку гомотопические кослои левых горизонтальных отображений естественно гомотопически эквивалентны. Гомотопический кослой среднего вертикального отображения будет $\Sigma^{1} \mathbb{L}\left(\pi_{1}(X \backslash Y) \rightarrow \pi_{1}(X)\right)$, как следует из диаграмм (6) и (23). Рассматривая гомотопические длинные точные последовательности в левом квадрате диаграммы (25), мы получаем диаграмму из утверждения теоремы.

Заметим, что точная последовательность (18) лежит в коммутативной диаграммe (22).

ТЕОРемА 3. Пусть $q \geqslant 3$ - коразмерность подмногообразия $Y$ в многообразии $X$ и $q^{\prime} \geqslant 3$ - коразмерность подмногообразия $Z$ в многообразии $Y$. Тогда имеет место изоморфизм

$$
L T_{n-q-q^{\prime}}(X, Y, Z)=L_{n}\left(\pi_{1}(X)\right) \oplus L_{n-q}\left(\pi_{1}(Y)\right) \oplus L_{n-q-q^{\prime}}\left(\pi_{1}(Z)\right) .
$$


ДоКАЗАТЕЛЬСтво. В соответствии с [1] мы имеем следующие изоморфизмы:

$$
L P_{n-q}(F) \cong L_{n}\left(\pi_{1}(X)\right) \oplus L_{n-q}\left(\pi_{1}(Y)\right)
$$

для $q \geqslant 3$ и

$$
\begin{aligned}
L P_{k}(\Psi) \cong & L_{n-q}\left(\pi_{1}(Y)\right) \oplus L_{k}\left(\pi_{1}(Z)\right), \\
& L S_{k}(\Psi) \cong L_{k}(Z)
\end{aligned}
$$

для $q^{\prime} \geqslant 3$. Для $q \geqslant 3$ мы еше имеем изоморфизм фундаментальных групп $C=\pi_{1}(X \backslash Y) \cong \pi_{1}(X)$. Используя диаграмму (6), мы можем записать часть диаграммы $(22)$ в следуюшей форме:

$$
\begin{aligned}
& L_{n}\left(\pi_{1}(X)\right) \quad \stackrel{\text { mono }}{\rightarrow} \quad L_{n}\left(\pi_{1}(X)\right) \oplus L_{n-q}\left(\pi_{1}(Y)\right)
\end{aligned}
$$

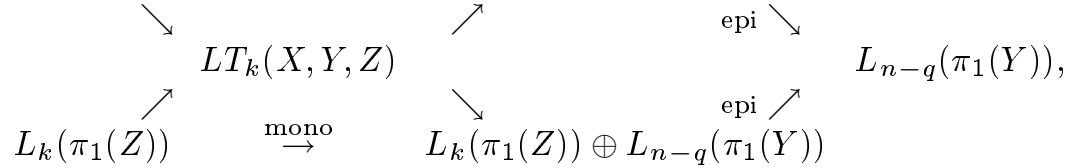

где $k=n-q-q^{\prime}$. В частности, диаграмма (26) содержит короткую точную последовательность

$$
0 \rightarrow L_{n}\left(\pi_{1}(X)\right) \rightarrow L T_{k}(X, Y, Z) \rightarrow L_{k}\left(\pi_{1}(Z)\right) \oplus L_{n-q}\left(\pi_{1}(Y)\right) \rightarrow 0
$$

Так как верхнее горизонтальное отображение в диаграмме (26) имеет левое обратное отображение и отображение

$$
L T_{k}(X, Y, Z) \rightarrow L_{n}\left(\pi_{1}(X)\right) \oplus L_{n-q}\left(\pi_{1}(Y)\right)
$$

эпиморфнн, то отображение $L_{n}\left(\pi_{1}(X)\right) \rightarrow L T_{k}(X, Y, Z)$ имеет левое обратное отображение. Это дает нужный изоморфизм.

Обозначим через $\mathbb{S}(X, Y, Z)$ гомотопический кослой отображения $\Theta$, полученного в теореме 1. Тогда гомотопические группы

$$
\mathscr{S}_{n}(X, Y, Z)=\pi_{n}(\mathbb{S}(X, Y, Z))
$$

будут естественньм обобщением структурных групп $\mathscr{S}_{n}(X)$ и $\mathscr{S}_{n}(X, Y, \xi)$. Из расслоения

$$
X_{+} \wedge \mathbf{L}_{\bullet} \stackrel{\Theta}{\longrightarrow} \Sigma^{q+q^{\prime}} \mathbb{L} T(X, Y, Z) \longrightarrow \mathbb{S}(X, Y, Z)
$$

мы получаем гомотопическую длинную точную последовательность

$$
\cdots \rightarrow L T_{k+1}(X, Y, Z) \rightarrow \mathscr{S}_{n+1}(X, Y, Z) \rightarrow H_{n}\left(X ; \mathbf{L}_{\bullet}\right) \rightarrow L T_{k}(X, Y, Z) \rightarrow \cdots
$$

где $k=n-q-q^{\prime}$ - размерность подмногообразия $Z$. Точная последовательность (27) для тройки многообразий $(X, Y, Z)$ обобщает как точную последовательность теории перестроек (2) для многообразия $X$, так и верхнюю точную последовательность в диаграмме (7) для пары многообразий $(X, Y)$. 
ТЕОРема 4. В приведенных выше обозначениях имеет место коммутативная диаграмма точных последовательностей

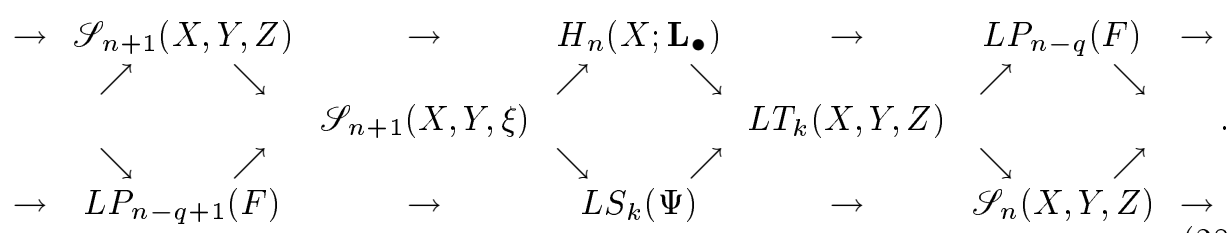

ДоказАТЕЛьство. Правый квадрат в диаграмме (19) является универсально-отталкивающим квадратом. Следовательно, кослои двух правых вертикальных отображений естественно гомотопически эквивалентны $\Sigma^{-q+1} \mathbb{S}(X, Y, Z)$. Теперь гомотопические длинные точные последовательности отображений, составляюших этот квадрат, дают диаграмму (26). Таким образом, теорема доказана.

Заметим, что диаграмма (28) является относительной версией диаграммы, описанной в [1; предложение 7.2 .6$, п. iv].

ТЕОРема 5. В приведенных выше обозначениях существует коммутативная диаграмма точных последовательностей

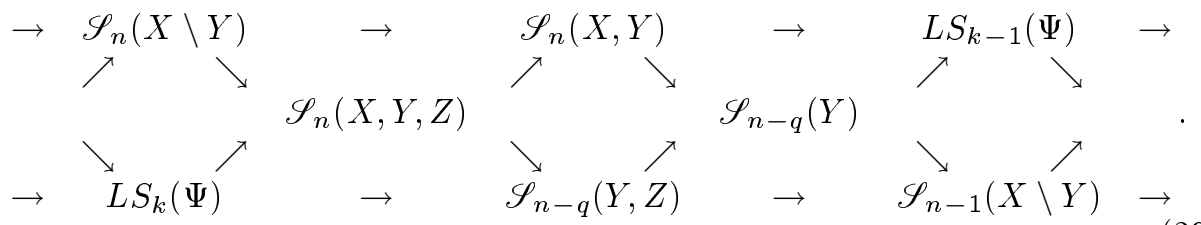

ДокАЗАТЕЛЬСтво. По лемме 2 мы можем записать коммутативный квадрат спектров

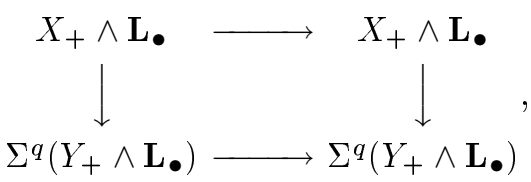

в котором горизонтальные отображения тождественны. Из диаграммы (25) следует гомотопически коммутативный квадрат спектров

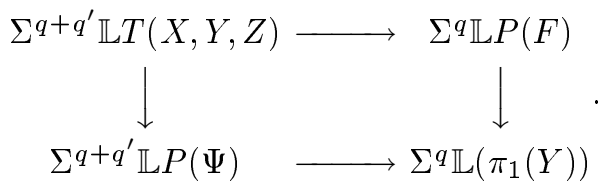

Рассмотрим естественные отображения спектров из диаграммы (30) в соответствующие спектры диаграммы (31), так что мы получаем гомотопически коммутативную диаграмму в форме куба. Коммутативность этой диаграммы следует из определения спектра $\mathbb{L} T(X, Y, Z)$ аналогично леммам 1 и 2. Квадраты (30) и (31) универсально-отталкивающие. Следовательно, кослои отображений спектров из 
квадрата (30) в спектры из квадрата (31) дают универсально-отталкивающий квадрат

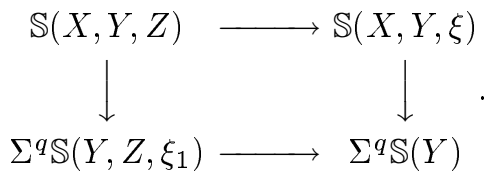

Гомотопические длинные точные последовательности отображений из универсально-отталкивающего квадрата (32) дают диаграмму (29).

СлЕДСТВИЕ. В приведенных выше обозначениях существует коммутативная диаграмма

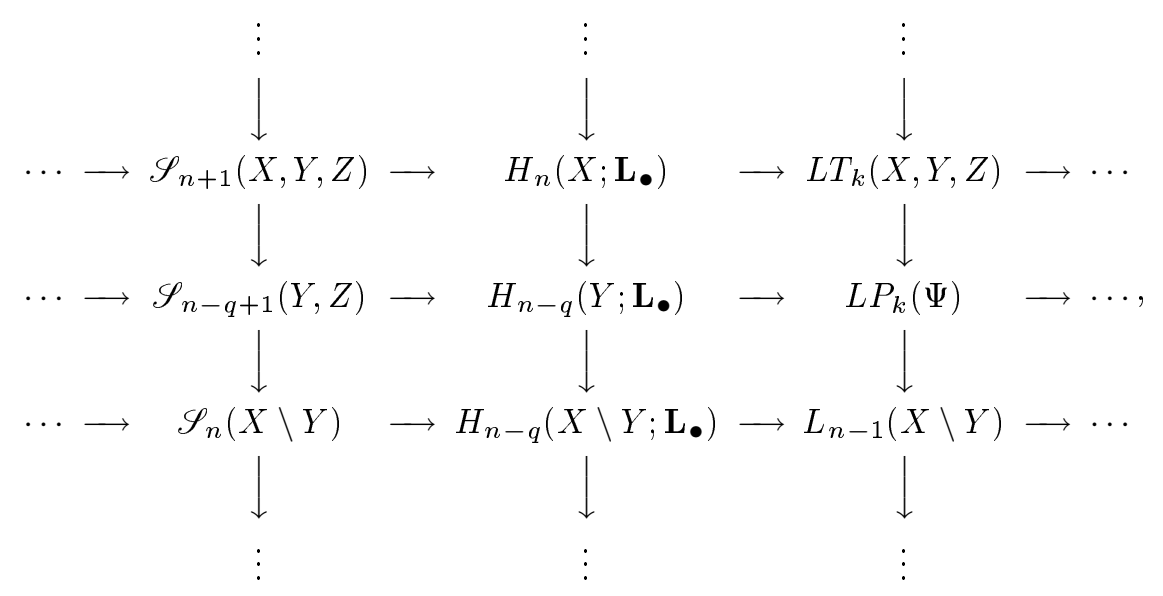

в которой строки и столбцы являются точными последовательностями.

ДокАЗАТЕльство. Гомотопические длинные точные последовательности отображений гомотопически коммутативного квадрата спектров

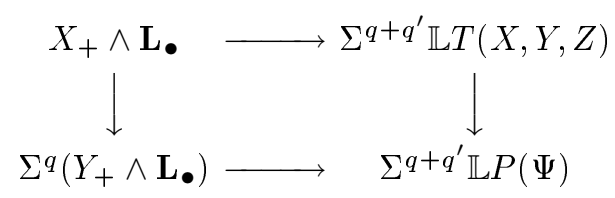

дают коммутативную диаграмму (33).

\section{§4. Применение к односторонним подмногообразиям}

В этом параграфе мы рассматриваем тройки многообразий $Z \subset Y \subset X$, где $Y \subset X$ и $Z \subset Y$ - пары Браудера-Ливси. В этом случае (см. [17] и [18]) $Y$ является односторонним подмногообразием коразмерности 1 в многообразии $X$, а $Z$ является односторонним подмногообразием коразмерности 1 в многообразии $Y$.

Квадрат $F$ фундаментальных групп, рассмотренный в $(3)$, имеет следуюшую форму:

$$
F=\left(\begin{array}{ccc}
\pi_{1}(\partial U) \longrightarrow & \pi_{1}(X \backslash Y) \\
\downarrow & \downarrow \\
\pi_{1}(Y) \longrightarrow & \pi_{1}(X)
\end{array}\right)=\left(\begin{array}{ccc}
A & \cong & \\
\downarrow i_{-} & & \downarrow \\
\downarrow i_{+} \\
B^{-} \stackrel{\cong}{\longrightarrow}
\end{array}\right)
$$


Все группы в квадрате (34) ориентированы. Более того, ориентация групшы $B^{-}$ отличается от ориентации группы $B^{+}$вне образов вертикальных отображений (которые являются вложениями индекса 2). Все отображения в квадрате $F(34)$, кроме нижнего горизонтального отображения, сохраняют ориентацию. Нижний изоморфизм сохраняет ориентацию на образе $i_{-}$и меняет ориентацию вне этого образа. В этом случае групшы препятствий к расшеплению $L S_{*}(F)$ называются группами Браудера-Ливси, обозначаемыми $L N_{*}\left(A \rightarrow B^{+}\right)=L N_{*}(A \rightarrow B)$. Мы имеем также изоморфизм [15]

$$
L P_{n}(F) \cong L_{n+1}\left(i_{-}^{*}\right)
$$

где $i^{*}: L_{n+1}\left(B^{-}\right) \rightarrow L_{n+1}(A)$ - отображение трансфера.

Квадрат $\Psi(15)$ фундаментальных групп для пары многообразий $Z \subset Y$ имеет вид

$$
\Psi=F^{-}=\left(\begin{array}{ccc}
A & \cong & A \\
\downarrow^{+} & & i_{-} \\
B^{+} \stackrel{\cong}{\longrightarrow} & B^{-}
\end{array}\right) .
$$

Квадрат (35) совпадает с квадратом (34) как коммутативный квадрат групп.

Аналогично $\S 3$ для тройки многообразий $(X, Y, Z)$ с парами Браудера-Ливси $(X, Y)$ и $(Y, Z)$ мы можем определить спектр $\mathbb{L} T(X, Y, Z)$ и групшы $L T_{*}(X, Y, Z)$ препятствий к перестройке тройки многообразий.

ПреДЛОЖЕНИЕ 2. Спектр $\mathbb{L} T(X, Y, Z)$ и группь $L T_{n}(X, Y, Z)$ функториально зависят от вложения $A \rightarrow B$ индекса 2 ориентированных групп и числа $n(\bmod 4)$.

ДокАЗАтЕльство. Рассмотрим в этом случае левьй универсально-отталкивающий квадрат

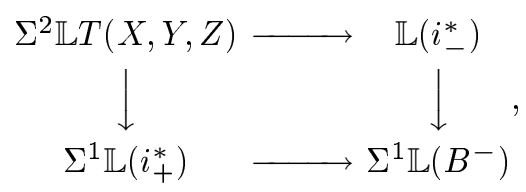

которьй возникает из диаграммы (25). Правое вертикальное отображение и нижнее горизонтальное отображение зависят функториально от вложения $A \rightarrow B$ ориентированных групп, поскольку реализация диаграммы (6) на уровне спектров функториальна (см. [15], [21]). Из этих отображений мы можем получить спектр $\Sigma^{2} \mathbb{L} T(X, Y, Z)$ в (36), используя конструкцию универсально-притягивающего квадрата, которая функториальна. Отсюда немедленно следует утверждение предложения.

По предложению 2 мы можем использовать следующие обозначения:

$$
\begin{gathered}
\mathbb{L} T(F)=\mathbb{L} T\left(F^{+}\right)=\mathbb{L} T(X, Y, Z), \\
L T_{*}(F)=L T_{*}\left(F^{+}\right)=L T_{*}(A \rightarrow B)=L T_{*}\left(A \rightarrow B^{+}\right)=L T_{*}(X, Y, Z)
\end{gathered}
$$

в рассматриваемом случае. 
ТЕОРЕма 6. В этих обозначениях существует коммутативная диаграмма точных последовательностей

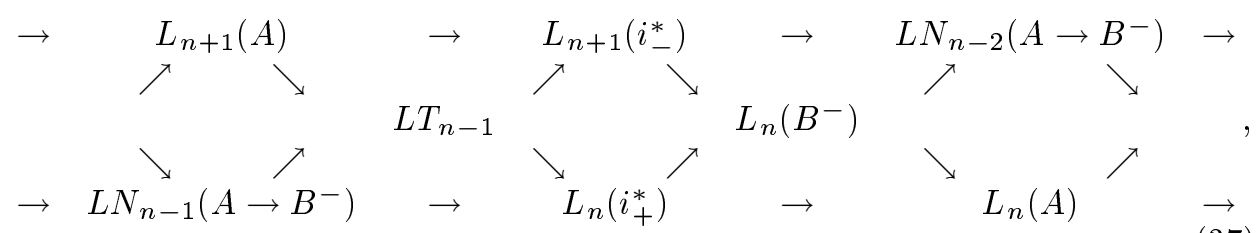

əде $L T_{n-1}=L T_{n-1}\left(A \rightarrow B^{+}\right)$.

ДокАЗАТЕльство.Действительно, этот результат является переформулировкой теоремы 2 в обозначениях этого параграфа.

В [8] была построена спектральная последовательность в теории перестроек. Основной шаг этой конструкции состоит в рассмотрении универсально-отталкивающих квадратов (23) и (24) для пар Браудера-Ливси $(X, Y)$ и $(Y, Z)$ соответственно. Затем мы можем рассмотреть фильтрацию спектров из [8]

$$
\cdots \rightarrow \mathbb{X}_{3,0} \rightarrow \mathbb{X}_{2,0} \rightarrow \mathbb{X}_{1,0} \rightarrow \mathbb{X}_{0,0} \rightarrow \mathbb{X}_{-1,0} \rightarrow \cdots
$$

которая была использована для построения вышеупомянутой спектральной последовательности. Здесь $\mathbb{X}_{0,0}=\mathbb{L}\left(B^{+}\right)$и $\mathbb{X}_{1,0}=\Sigma \mathbb{L} P(F)=\mathbb{L}\left(i_{-}^{*}\right)$. В соответствии с [8] спектр $\mathbb{X}_{2,0}$ получается использованием конструкции универсально-притягивающего квадрата для отображений спектров из (36)

$$
\mathbb{L}\left(i_{-}^{*}\right) \rightarrow \Sigma^{1} \mathbb{L}\left(B^{-}\right) \leftarrow \Sigma^{1} \mathbb{L}\left(i_{+}^{*}\right) .
$$

Другие спектры $\mathbb{X}_{k, 0}$ получаются итерацией этого процесса (см. [8]).

Согласно [8] мы имеем изоморфизмы

$$
E_{1}^{p, s}=\pi_{s-p}\left(\mathbb{X}_{p, 0}, \mathbb{X}_{p+1,0}\right)=L N_{s+2}(A \rightarrow B),
$$

а первьй дифференпиал

$$
d_{1}^{p, s}: E_{1}^{p, s} \rightarrow E_{1}^{p+1, s}
$$

совпадает с композицией

$$
L N_{s-2 p-2}\left(A \rightarrow B^{(-)^{p}}\right) \rightarrow L_{s-2 p-2}\left(B^{(-)^{p+1}}\right) \rightarrow L N_{s-2 p}\left(A \rightarrow B^{(-)^{p+1}}\right) .
$$

Отображения в диаграмме (40) возникают из диаграммы (6), рассматриваемой для случаев односторонних подмногообразий с квадратами $F(34)$ и $F^{-}(35)$.

Tеорема 7. Спектр $\mathbb{X}_{2,0}$ фильтрации (38) совпадает со спектром $\Sigma^{2} \mathbb{L} T(F)$, определенным в этом параграфе для задачи перестройки тройки многообразий. Отображсние $\mathbb{X}_{2,0} \rightarrow \mathbb{X}_{1,0}$ фильтрации (38) совпадает с отображсением

$$
\Sigma^{2} \mathbb{L} T(A \rightarrow B) \rightarrow \mathbb{L}\left(i_{-}^{*}\right)=\Sigma^{1} \mathbb{L} P(F)
$$

из диаграмми (36). 
ДокАЗАТЕЛЬство. В соответствии с (39) определение спектра $\mathbb{X}_{2,0}$ совпадает с определением спектра $\Sigma^{2} \mathbb{L} T(F)$ посредством универсально-притягивающего квадрата (36).

ЗАмЕчАНИЕ 1. С алгебраической точки зрения мы можем построить спектр $\mathbb{L} T\left(A \rightarrow B^{+}\right)$по вложению $A \rightarrow B^{+}$индекса 2 ориентированных групп. Фактически имеется реализация алгебраической версии диаграммы (6) на уровне спектров (см. [11], [12], [15]). В частности, мы можем определить спектр $\mathbb{L} T\left(A \rightarrow B^{-}\right)$, входящий в следующий универсально-притягивающий квадрат спектров:

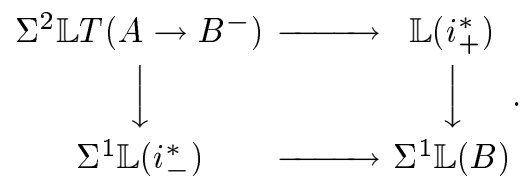

Тог да групшы препятствий $L T_{*}\left(A \rightarrow B^{-}\right)=\pi_{*}\left(\mathbb{L} T\left(A \rightarrow B^{-}\right)\right)$определены. Гомотопические длинные точные последовательности квадрата (41) дают следующую коммутативную диаграмму:

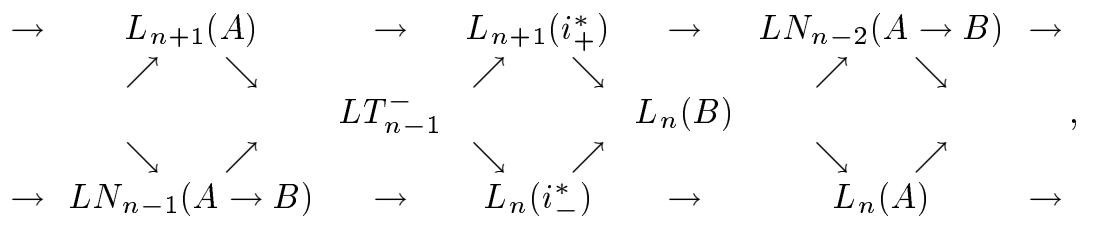

где $L T_{n-1}^{-}=L T_{n-1}\left(A \rightarrow B^{-}\right)$. Групшы $L T_{*}\left(A \rightarrow B^{-}\right)$отличаются от групп $L T_{*}\left(A \rightarrow B^{+}\right)$в обшем случае. В действительности можно перенести все результаты этого параграф̆а на случай квадратичного расширения антиструктур и $L$-групп с декорациями (см. [6] и [15]).

ПРЕДЛОЖЕНИЕ 3. Спектр $\mathbb{X}_{3,0}$ фильтрации (38) вХодит в следующий универсально-притягивающий квадрат спектров:

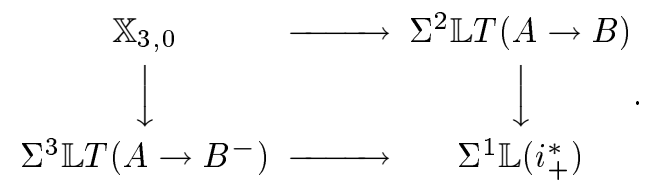

ДокАЗАтЕльство. Результат немедленно следует из рассмотрения универсально-притягивающих квадратов (36) и (41) и определения фильтрации (38) в $[8]$.

ПрИмеР 1. Рассмотрим тройку действительных проективных пространств $\mathbb{R P}^{2 l-1} \subset \mathbb{R P}^{2 l} \subset \mathbb{R P}^{2 l+1}, 2 l-1 \geqslant 3$. Мы имеем $\pi_{1}\left(\mathbb{R P}^{k}\right)=\mathbb{Z} / 2$ для любого $k \geqslant 2$. Гомоморфизм ориентации $w: \mathbb{Z} / 2 \rightarrow\{ \pm 1\}$ тривиален для нечетного $k$ и является изоморфизмом для четного $k$. Все группы $L_{n}(1), L_{n}\left(\mathbb{Z} / 2^{+}\right)$и $L_{n}\left(\mathbb{Z} / 2^{-}\right)$ хорошо известны [3], и имеют место изоморфизмы $L N_{*}\left(1 \rightarrow \mathbb{Z} / 2^{+}\right) \cong L_{*+2}(1)$ и $L N_{*}\left(1 \rightarrow \mathbb{Z} / 2^{-}\right) \cong L_{*}(1)$. Используя диаграммньй поиск в диаграмме (6) для вложений $1 \rightarrow \mathbb{Z} / 2^{+}$и $1 \rightarrow \mathbb{Z} / 2^{-}$, можно вычислить соответствующие группы препятствий к перестройкам для пар многообразий. Мы получаем

$$
\begin{aligned}
L P_{n}(F) & =L_{n+1}\left(i_{-}^{*}\right)=\mathbb{Z} / 2, \mathbb{Z} / 2, \mathbb{Z} / 2, \mathbb{Z}, \\
L P_{n}\left(F^{-}\right) & =L_{n+1}\left(i_{+}^{*}\right)=\mathbb{Z}, \mathbb{Z} / 2, \mathbb{Z} / 2, \mathbb{Z} / 2
\end{aligned}
$$

для $n=0,1,2,3(\bmod 4)$ соответственно. 
ПРЕДЛОЖЕНИЕ 4. Пусть $M^{m}$ - топологическое многообразие размерности $m$ с тривиальной фундаментальной группой. Пусть $n=m+2 l-1 \geqslant 5$. Для тройки многообразий

$$
\mathbb{R P}^{2 l-1} \times M \subset \mathbb{R P}^{2 l} \times M \subset \mathbb{R P}^{2 l+1} \times M
$$

с $l \geqslant 2$ группьц $L T_{n}$ изоморфнь

$$
\mathbb{Z} \oplus \mathbb{Z} / 2, \quad \mathbb{Z} / 2, \quad \mathbb{Z} \oplus \mathbb{Z} / 2, \quad \mathbb{Z} / 2
$$

для $n=0,1,2,3(\bmod 4)$ соответственно. Более того, естественние забивающие отображсения $L T_{n}(F) \rightarrow L P_{n}\left(F^{-}\right)$u $L T_{n}(F) \rightarrow L P_{n+1}(F)$ являются эпиморфизмами для всех $n$.

ДокАЗАтЕльство. Диаграммный поиск в диаграмме (37) дает результат.

ПримеР 2. Рассмотрим тройку действительных проективных пространств $\mathbb{R P}^{2 l} \subset \mathbb{R P}^{2 l+1} \subset \mathbb{R P}^{2 l+2} \mathrm{c} l \geqslant 1$. Аналогично примеру 1 мы получаем следующий результат.

ПРЕДЛОЖЕНИЕ 5. Пусть $M^{m}$ - топологическое многообразие размерности $m$ с тривиальной фундаментальной группой. Пусть $n=m+2 l \geqslant 5$. Для тройки многообразий

$$
\mathbb{R P}^{2 l} \times M \subset \mathbb{R P}^{2 l+1} \times M \subset \mathbb{R P}^{2 l+2} \times M
$$

мъи имеем изоморфизмь $L T_{0}^{-} \cong \mathbb{Z} / 2 \oplus \mathbb{Z} / 2$ и LT $T_{1}^{-} \cong \mathbb{Z} / 2$. Группьи $L T_{3}^{-}$и $L T_{2}^{-}$ входят в следуюшую точную последовательность:

$$
0 \rightarrow L T_{3}^{-} \rightarrow \mathbb{Z} \rightarrow \mathbb{Z} \rightarrow L T_{2}^{-} \rightarrow \mathbb{Z} / 2 \rightarrow 0
$$

ДокАЗАТЕльство. Диаграммный поиск в диаграмме (42) дает результат.

\section{Список литературы}

1. Ranicki A. A. Exact sequences in the algebraic theory of surgery. Princeton: Princeton Univ. Press, 1981.

2. Ranicki A. A. Algebraic $L$-theory and topological manifolds. Cambridge: Cambridge Univ . Press, 1992.

3. Wall C. T. C. Surgery on compact manifolds. Providence, R.I.: Amer. Math. Soc., 1999.

4. Ranicki A. A. The total surgery obstruction // Lecture Notes in Math. 1979. V. 763. P. $275-316$.

5. Ferry S. C., Ranicki A. A., Rosenberg J. (Eds.) Novikov conjectures, index theorems and rigidity. V. 1. Cambridge: Cambridge Univ. Press, 1995. (London Math. Soc. Lecture Note Ser. V. 226.)

6. Cavicchioli A., Muranov Yu. V., Repovš D. Algebraic properties of decorated splitting obstruction groups // Boll. Unione Mat. Ital. Sez. B Artic. Ric. Mat. (8). 2001. V. 4. P. 647-675.

7. Hambleton I. Projective surgery obstructions on closed manifolds // Lecture Notes in Math. 1982. V. 967. P. 101-131.

8. Хәмблтон И., Харииладзе А. Ф. Спектральная последовательность в теории перестроек // Матем. сб. 1992. Т. 183. №9. С. 3-14.

9. Муранов Ю. В. Задача расщепления // Труды МИРАН. 1996. Т. 212. С. 123-146. 
10. Ахметьев П. М., Муранов Ю. В. Препятствия к расщеплению многообразий с бесконечной фундаментальной группой // Матем. заметки. 1996. Т. 60. С. 163-175.

11. Муранов Ю. В. Группы препятствий к расщеплению и квадратичные расширения антиструктур // Изв. РАН. Сер. матем. 1995. Т. 59. С. 107-132.

12. Муранов Ю. В., Хәмблтон И. Проективныегрупшы препятствий к расщеплению вдоль односторонних подмногообразий // Матем. сб. 1999. Т. 190. № 10. С. 65-86.

13. Муранов Ю.В., Харииладзе А.Ф. Группы Браудера-Ливси абелевых 2-групп // Матем. сб. 1990. Т. 181. № 8. С. 1061-1098.

14. Муранов Ю. В., Репови Д. Группы препятствий к перестройкам и расщеплениям для пары многообразий // Матем. сб. 1997. Т. 188. № 3. С. 127-142.

15. Ranicki A. A. The $L$-theory of twisted quadratic extensions // Canad. J. Math. 1987. V. 39. P. $345-364$.

16. Ruini B., Spaggiari F. On the computation of $L$-groups and natural maps // to appear.

17. Browder W., Livesay G. R. Fixed point free involutions on homotopy spheres // Bull. Amer. Math. Soc. (N.S.). 1967. V. 73. P. 242-245.

18. Cappell S. E., Shaneson J. L. Pseudo-free actions // Lecture Notes in Math. 1979. V. 763. P. $395-447$.

19. Hambleton I., Pedersen E. Topological equivalences of linear representations for cyclic groups // MPI Preprint, 1997.

20. Jimenez R., Muranov Yu. V. Surgery transfer maps for triples of manifolds // Publications Preliminaries del Instituto de Matematicas-Cuernavaca. 2002. № 721 .

21. Hambleton I., Ranicki A., Taylor L. Round L-theory // J. Pure Appl. Algebra. 1987. V. 47. Р. $131-154$.

22. Малешич Й., Муранов Ю.В., Реповш Д. Групшы препятствий к расщеплению в коразмерности 2 // Матем. заметки. 2001. Т. 69. № 1. С. 52-73.

23. Lück W., Ranicki A.A. Surgery transfer // Lecture Notes in Math. 1988. V. 1361. P. $167-246$.

24. Lück W., Ranicki A.A. Surgery obstructions of fiber bundles // J. Pure Appl. Algebra. 1992. V. 81. P. 139-189.

25. Switzer R. Algebraic topology - homotopy and homology. Berlin: Springer-Verlag, 1975.

Институт современных знаний,

Витебск, Беларусь;

Institute for Mathematics, Physics and Mechanics,

University of Ljubljana, Ljubljana, Slovenia;

Dipartimento di Matematica, Università di Modena e Reggio E.,

Modena, Italy

E-mail: ymuranov@imk.edu.by

dusan.repovs@uni-lj.si

spaggiari@unimo.it
Поступила в редакцию

11.07.2002 